\title{
Diagnostic and prognostic molecular markers in hepatocellular carcinoma
}

\author{
Beatriz Mínguez ${ }^{\mathrm{a}, \mathrm{b}, *}$ and Anja Lachenmayer ${ }^{\mathrm{c}, \mathrm{d}}$ \\ ${ }^{a}$ Liver Unit, Department of Internal Medicine, Hospital Universitari Vall d'Hebrón, Barcelona, Spain \\ ${ }^{\mathrm{b}}$ Centro de Investigación Biomédica en Red de Enfermedades Hepáticas y Digestivas (CIBEREHD), Instituto \\ Carlos III, Spain \\ ${ }^{c}$ Liver Cancer Program, Division of Liver Diseases, Mount Sinai School of Medicine, New York, NY, USA \\ ${ }^{\mathrm{d}}$ Department of General-, Visceral- and Pediatric Surgery, University Hospital Düsseldorf, Düsseldorf, Germany
}

\begin{abstract}
Hepatocellular carcinoma (HCC) is one of the most lethal cancers worldwide, representing also the main cause of death among cirrhotic patients. In contrast to most other solid tumors, the underlying cirrhotic liver disease in HCC patients greatly impairs tumor related prognosis, conferring this neoplasm a unique situation, in which accurate prognostic prediction is a relevant and unmet need.

Although clinical staging systems have improved significantly and now comprise tumor characteristics, liver function and patient performance status, the integration of molecular data into these algorithms is still hypothetical.

Molecular profiling of HCC has led to a better understanding of the physiopathology of this neoplasm and has allowed developing novel therapeutic approaches (e.g. molecular targeted therapies) for a tumor previously considered as therapy-refractory.

Integrative analysis of different reported genomic datasets has revealed common subclasses between different studies, highlighting their biological relevance in HCC. Gene signatures derived from tumors and from the adjacent tissue have been able to differentiate subclasses with different outcomes and have been proposed as potential predictive markers in the clinical setting. Genomic characterization of surrounding non-tumor tissue might be of particular interest to identify patients at high risk of developing HCC and therefore to select those patients that would benefit of potential chemopreventive strategies.

Epigenetic analyses (methylation and miRNA profiling) are adding up to the knowlegde derived from gene expression data and should not be forgotten in the molecular diagnosis of HCC.

Integrative analyses of genetic and epigenetic information of the tumor and the surrounding tissue should be used to identify novel biomarkers and therapeutic targets in $\mathrm{HCC}$, to improve existing treatment algorithms and to eventually design a more personalized medicine in this devastating disease.
\end{abstract}

Keywords: Liver cancer, genomic profiling, personalized medicine, prognostic modelling, biomarkers, gene signatures, miRNA, epigenetics

\section{Introduction}

Hepatocellular carcinoma (HCC) is not only the sixth most common cancer and the third cause of cancerrelated mortality worldwide, but also the leading cause

\footnotetext{
*Corresponding author: Beatriz Mínguez, MD, Liver Unit, Department of Internal Medicine, Hospital Universitario Vall d'Hebron, Psg. Vall d'Hebrón 119-129, Barcelona, 08035, Spain. Tel.: +34 93 27461 40, (Ext 6554); E-mail: bminguez@vhebron.net.
}

of death among cirrhotic patients [1,2]. Cirrhosis is the underlying liver disease in eighty percent of HCCs, adding a very distinctive feature to this tumor compared to other solid neoplasms.

Besides the high prevalence of hepatitis $\mathrm{C}$ virus (HCV) infection as the main reason for the increasing incidence of HCC in Western countries [3], multiple etiologies have been identified leading to liver damage and to increased incidence of HCC (chronic viral hepatitis $\mathrm{B}$, alcohol consumption and alfatoxin among oth- 
ers) [4]. This confers HCC not only a more complex clinical approach but also a high molecular variability.

Although surveillance programs for cirrhotic patients have improved significantly in the past years [5], only one third of HCC patients are diagnosed at early stages, when they are still eligible for potentially curative therapies as resection, transplantation or local ablation [4]. Among these early tumors, one fifth present with very aggressive behaviour, rapid dissemination and poor survival. Since this subgroup of patients cannot be identified with the currently used clinical staging system, other prognostic risk assessments like genomebased classification systems have been proposed to better evaluate the patients before treatment [6].

All patients with advanced stages of the disease have very limited survival expectancy and so far only the multikinase inhibitor sorafenib has demonstrated to improve survival rates in this setting [7]. Molecular data generated from tumors responding to Sorafenib or other potential therapies could help to identify patients whom benefit most of each treatment and could be a valuable tool in clinical practice of HCC.

However, molecular data of any kind is still not integrated in the therapeutic decision-making algorithm, and in the daily clinical management of HCC, different staging systems including tumor characteristics (tumor size, number of nodules, vascular invasion, etc.) and markers for severity of the underlying liver disease (e.g. Child Pugh score) are used to classify patients, to predict outcome and to suggest the appropriate treatment option [4].

This review aims to describe the relevance of genomics as a novel diagnostic and prognostic tool in HCC, to give an overview on epigenetics in HCC (miRNA, methylation, etc.) and to discuss how integration of this molecular data into the existing clinical algorithms might improve the accuracy of prognosis prediction and might lead to a more efficient personalized medicine in HCC.

\section{Molecular diagnosis of $\mathrm{HCC}$}

Implementation of surveillance programs for patients at risk of developing HCC together with application of advanced imaging techniques is changing the prognostic landscape of this neoplasm and allows the diagnosis of patients at earlier stages.

American Association for the Study of Liver Diseases (AASLD) guidelines propose a non-invasive diagnostic algorithm that is applicable for cirrhotic pa- tients and for most of the HCCs above $2 \mathrm{~cm}$ in diameter as well as for around $30 \%$ of the tumors less than $2 \mathrm{~cm}[5]$.

Although tumor biopsy should be performed when non-invasive diagnosis is not feasible, there is a high false negative rate for small nodules. Differential diagnosis between dysplastic nodule and early HCC is very complicated even for experienced liver pathologists, because stromal invasion, a typical malignant feature, could be absent in the biopsy specimen [8]. Accurate diagnosis of these small nodules is very important, because aggressive treatments should be applied in the malignant setting, whereas dysplastic nodules should only be closely followed by imaging techniques.

Alpha-fetoprotein (AFP) has been used as a serum marker for HCC for many years, although some patients with HCC might present without any serum elevation. On the contrary patients with cirrhosis can have increased AFP serum levels in the absence of a tumor. Although tested in multiple clinical studies, no true benefit of AFP as a maker for surveillance programs could be detected [9]. However, AFP seems to have prognostic value if highly elevated at the time of tumor diagnosis, and could be use as a surveillance marker for tumor progression after treatment in patients with AFP-producing HCC [10]. Other reported serum HCC markers, as lens culinaris agglutinin-reactive AFP (AFP-L3), des-gamma carboxyprotrombin (DCP) and glypican-3 (GPC3) have not shown better performance in early detection of HCC [11].

Based on the information obtained from molecular profiling, additional diagnostic tissue biomarkers have been prospectively tested by immunostaining for early HCC. Glypican-3 (GPC3), a member of the glypican family of glycosyl-phosphatidylinositol-anchored cellsurface heparin sulphate proteoglycans, arises in many studies as a potential marker of malignancy [12] and reaches sensitivity of $77 \%$ and specificity of $96 \%$ to diagnose early small $\mathrm{HCC}$ versus high grade dysplastic nodule (HGDN) [13]. Combination of GPC3 with Heat shock protein 70 (HSP70) (implicated in tumorigenesis by regulation of cell cycle progression and apoptosis) and Glutamine synthetase (GS) (target gene of $\beta$-catenin) has been described and validated reaching a sensitivity and specificity of $72 \%$ and $100 \%$, respectively. This panel of stainings is often used to confirm or to resolve complicated cases of histopathological diagnosis $[8,13,14]$.

Despite the large number of studies dedicated to molecular diagnosis of HCC, truly reliable biomarkers for this neoplasm still need to be identified. Merging 
clinical algorithms and potential molecular diagnostic and prognostic markers is an unmet need awaited to improve accuracy and applicability of the current guidelines.

\section{Lessons learned from gene expression profiling of HCC: molecular classification and common molecular subclasses of HCC}

The main purpose of a correct classification in HCC is to predict outcome, select the optimal treatment for each patient and to provide an adequate scenario to design clinical trials in which patients with similar characteristics can be properly compared. Among the clinical classifications in practice, the Barcelona Clinic Liver Cancer (BCLC) staging system addresses not only tumor burden variables but also stage of the underlying liver disease, a life-threatening condition with clear prognostic implications. Subsequently the EASL (European Association for the Study of the Liver) and the AASLD recommend using the BCLC system as the standard guideline for the clinical management of HCC patients $[5,15]$. While several clinical staging systems have been proposed for HCC, not a single one has intended to include molecular parameters from the quickly accumulating genomic studies identifying molecular classifications in this malignancy.

Novel high throughput technologies such as gene expression microarray and SNP-array facilitate the identification of molecular classes in HCC and lead to an exponential increase of conducted genomic studies. But despite several different proposed classes in different cohorts of patients (e.g. distinct in etiology, tumor stage or country of origin), no ultimate molecular classification has been described so far. However, some of these studies were able to overlap molecular classes of different studies and identified homogeneous subgroups of patients based exclusively on molecular parameters [32].

One interesting meta-analysis enrolled a total of 603 HCC patients from different continents and revealed 3 common transcriptome-based subclasses, despite the clinical and etiological heterogeneity of the patients included [32]. The study analyzed 9 independent datasets and described three subclasses, clearly unravelling aggressive and less-aggressive tumors. Remarkably, gene-expression pattern showed a significant similarity with previously reported subclasses of aggressive and less-aggressive tumors as shown in Fig. 1.
Notably, aggressive tumors clustered in subclasses $\mathrm{S} 1$ and S2 and were associated with clinical characteristics like larger tumor size and poor histological differentiation. HBV-related HCCs were enriched in these subgroups, possibly related to the HBV carcinogenic effect independent of the development of liver cirrhosis and frequently related to rapid progression [25,37].

The subclass S1 was characterized by activation of the Wnt pathway, most likely augmented by activation of the TGF-beta pathway. Although the activation was validated by immunostaining showing increased levels of cytoplasmic $\beta$-Catenin, no correlation to known CTNNB1 mutations could be discovered, further highlighting TGF $\beta$-Wnt interactions in this subclass.

S2 was characterized by Akt and Myc pathway activation as well as down-regulation of IFN-target genes. Overexpression of AFP nicely correlated to elevated AFP serum levels of the same patients in this class.

S3 was defined as the subclass of less-aggressive tumors with histologic evidence of better differentiation and a gene expression profile exhibiting relatively higher levels of expression of hepatocyte function-related genes. GSEA also revealed differential activation of p53 and p21 target gene sets in S3 tumors, validated by significantly lesser nuclear p53 staining compared with $\mathrm{S} 1$ and $\mathrm{S} 2$. The previously reported less aggressive subclasses, as CTNNB1, polysomy of chromosome 7 [26] and Boyault's G5,6 [25], were also found to be associated to $\mathrm{S} 3$.

In addition, 2 recent genomics studies suggested that gene expression profiles of the surrounding nontumor liver tissue contain important prognostic information. This observation highlights the so called "field effect" $[21,23]$ and such molecular profiles are able to identify patients at risk of early recurrence or of developing de novo $\mathrm{HCC}$ respectively. The adjacent tissue gene signatures most likely contain information of the liver function and the microenvironment conditions that eventually facilitate intrahepatic tumor-cell dissemination or neo-tumorigenesis, but need to be further explored and validated.

Molecular information of the tumor and the surrounding tissue can not only be used to classify malignancies, but also to generate outcome related gene signatures. While all reported molecular classifications of HCC have been exhaustively reviewed elsewhere [16], Table 1 overviews gene signatures with prognostic implications previously described in HCC.

The current clinical staging systems for HCC should be refined with molecular and genomic variables in order to capture all prognostic information available. 


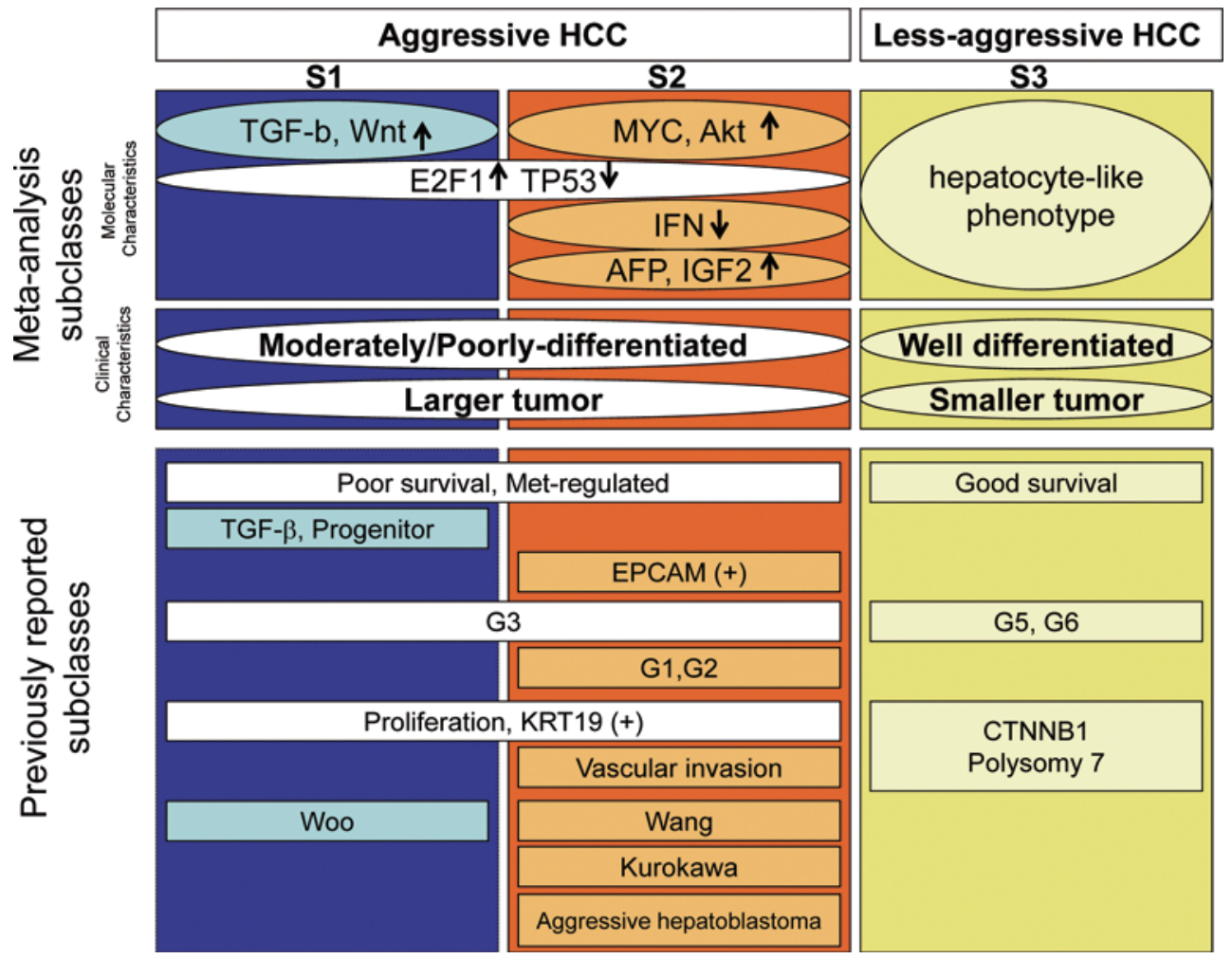

Fig. 1. Transcriptome based common molecular classification of HCC. A meta-analysis [17] including 603 HCC patients from 9 independent datasets defined three common transcriptome-based subclasses, characterized by molecular and clinical features. The lower part of the graphic describes signatures and subclasses previously reported [17-20,25,26,28,33-36] and their correlation to subclasses S1, 2 and 3. Modified from Hoshida et al. [17].

Future staging systems should probably include clinical parameters (tumor characteristics and liver function) and genomic data obtained from the tumor as well as from the adjacent non-tumoral tissue [38]. Molecular information from the adjacent tissue might be more relevant in early cases, where the tumor can be completely resected or ablated and the field effect will play a pivotal role in de novo tumor development. When the tumor progresses, it might be more important to obtain molecular and genomic data from the tumor itself as this will most likely condition the patient's survival.

\section{Additional information to the molecular} classification of HCC: miRNA and epigenetics. Role in hepatocarcinogenesis and prognostic value

MicroRNAs (miRNAs) are small noncoding RNAs that negatively regulate the expression of target mRNA transcripts at a post-transcriptional level [39]. They have been shown to be involved in a variety of cellular processes such as cell proliferation, cell differentiation, apoptosis, neuronal patterning and stem cell maintenance [40]. miRNAs can act as tumor suppressor genes or oncogenes depending on their target genes $[41,42]$ and are frequently dysregulated in cancer.

A recent study supported the value of miRNA expression profiling for cancer classification by showing that the tissue lineage-specific information classified samples more accurately than mRNA profiling did [43].

miRNA profiles could also be considered as potential biomarkers for early cancer diagnosis given its ability to distinguish cancer from normal tissues [41]. Unsupervised clustering of miRNA profiles revealed subclasses that match and add information to previously described molecular subclasses identified in a cohort of HCV-related HCCs [44].

Prognostic prediction is critical in cancer and many studies have proposed that miRNA profiling could be useful to classify cancer patients according to their clinical outcome [45-47]. This has been also described in HCC (i.e., downregulation of miR 26-a or 20miRNA signature are related to poor outcomes, whereas upreg- 
Table 1

\begin{tabular}{ll} 
Molecular gene signatures with prognostic implications described in HCC \\
\hline & Outcome implication \\
\hline Signatures related to survival & \\
Cluster A/ Hepatoblastoma signature [17-20] & Poor survival \\
Late recurrence, Poor prognosis* [21] & Poor survival \\
EPCAM [22] & Poor survival \\
Intrahepatic metastasis* [23] & Recurrence, poor survival \\
Cluster A [24] & Poor survival \\
G3 [25] & Poor survival \\
Signatures related to recurrence & \\
Polysomy 7 [26] & \\
Kurokawa [27] & Early recurrence \\
Woo [28] & Early recurrence \\
Wang [29] & Early recurrence \\
Yoshioka [30] & Recurrence \\
Iizuka [31] & Early recurrence \\
\hline
\end{tabular}

* molecular information from gene expression profiling of the non tumoral adjacent tissue.

ulation of miR 125-a is associated to better survival). Table 2 resumes all known miRNA dysregulations in HCC.

Among the overexpressed miRNAs in HCC, miR21 has been shown to negatively down-regulate the expression of the tumor suppressor gene PTEN, leading to activation of PI3K/AKT pathway [56]. miR221 overexpression has been reported to target cyclin G1 [57] and was associated to tumor multinodularity and higher recurrence [48]. Overexpression of miR143 was associated with metastasis [50], and overexpression of miR125B expression was correlated with good survival [49].

On the contrary downregulation of several miRNAs has been described for HCC. For example, Let-7 family members known to target oncogenes like MYC, RAS, and HMGA2 have been found significantly downregulated and associated to advanced stage HCC and poor prognosis [51]. miR122 is commonly down-regulated in HCC and is also related to poor prognosis [53], probably in relation to the loss of this hepatic specific miRNA and dedifferentiation of the tumor. Low expression of miR26 level has been proposed as an independent predictor of survival in HCC [52] and multiple miRNA expression based signatures related to prognosis have been reported recently [54,55].

miRNA profiling is expected as a source of additional information to better understand the complex molecular heterogeneity and to consequently provide the rationale for new therapeutic targets in HCC. Modulation of the expression of miR26A in preclinical models of $\mathrm{HCC}$ reduced cancer cell proliferation, induced tumor-specific apoptosis, and significantly delayed disease progression [58], suggesting that miRNA targeting is a feasi- ble therapeutic alternative in HCC. In fact, manipulation of expression of specific miRNAs by antagomirs (cholesterol-modified antisense oligonucleotides) and locked nucleic acids (LNA) are currently under development in vivo $[59,60]$.

Epigenetic alterations are heritable changes in the pattern of gene expression mediated by mechanisms different than alterations in the genomic DNA sequence. They modify transcription via potentially reversible mechanisms like DNA methylation, histone modifications and nucleosome remodelling. There is increasing evidence of their critical role in the development and progression of cancer, becoming almost an equivalent counterpart to the genetic alterations known in cancer [61].

Genome wide hypomethylation is a frequent event in cancer leading to genomic instability, which has also been described for HCC, associated to higher histological grade and larger size of the tumors $[62,63]$. Also, hypomethylation of promoter regions leading to reactivation of oncogenes (c-myc or RAS) was demonstrated in HCC $[62,64]$.

Hypermethylation of $\mathrm{CpG}$ islands within the regulatory regions of genes usually leads to gene silencing, as reported for the tumor suppressor genes p16, RASSF1, IGFBP3, E-cadherin and SOCS1 in HCC $[16,65]$. Table 3 is giving a more detailed overview on all known epigenetic changes in HCC published so far.

Also the enzymes responsible for DNA methylation (DNA methyltransferases ((DNMTs)) are known to be overexpressed in several cancers and have been shown to be associated to poor survival in HCC $[66,67]$. Even $\mathrm{HCV}$ infection has been related to acceleration of the methylation process in HCC [68] and needs further in- 
Table 2

Dysregulated microRNA in HCC

\begin{tabular}{lll}
\hline Expression of miRNA & microRNA & \multicolumn{1}{c}{ Clinical relevance } \\
\hline Up-regulated & miRNA 221 & Multinodularity, recurrence [48] \\
& miRNA 125a & Good survival [49] \\
& miRNA 143 & Metastasis [50] \\
& miRNA 224 & \\
& miRNA 9 & \\
& miRNA 18 & \\
& miRNA 181 & \\
miRNA 21 & Early recurrence, poor prognosis [51] \\
& let-7 family & Poor survival [52] \\
& miRNA 26 & Poor survival [53] \\
& miRNA 122 & \\
& miRNA 1 & \\
& miRNA 124 & \\
& miRNA 203 & \\
& miRNA 195 & \\
& miMIR 101 & \\
& miRNA 34A & \\
& miRNA 125A & \\
& miRNA 199A,B & \\
& miRNA 200A & Metastasis, poor survival [54] \\
\hline miRNA signatures & 20-miRNA signature & Poor survival [55] \\
& 19-miRNA signature & \\
& Up-regulation of 4 miRNA & Poor differentiation [41] \\
\hline
\end{tabular}

vestigation to fully understand its importance in hepatocarcinogenesis.

Histone modifications are more complex alterations on the N-terminal tails (methylation, acetylation, ubiquination, etc.) that can regulate gene expression either directly or through chromatin remodelling. Nevertheless, only very few genes regulated by histone modifications have been described in HCC so far (Table 3) $[65,69,70]$.

DNA-methylation profiling has exposed specific profiles of hypermethylated $\mathrm{CpG}$ islands (hypermethylomes) that were able to distinguish between different tumor types [71] and to predict patient outcome. Different profiles have been reported as associated with clinical characteristics and outcomes i.e. genome wide hypomethylation and hypermethylation of Ecadherin have been related to tumor progression and poor survival $[62,72]$. Table 4 summarizes epigenetic alterations with prognostic implications described in HCC. These results suggest that large-scale and whole genome DNA methylation profiling may be used to classify HCC tumors, as it has been reported recently [73].

Interestingly, a recent study has shown that a methylation profile generated from the adjacent liver tissue of the surgical margins of resected HCC patients was clearly related to survival, suggesting the importance of microenviroment in hepatocarcinogenesis and high- lighting again the prognostic value of epigenetics in HCC [74].

These potentially reversible epigenetic alterations display a great target for cancer therapy [75] and several demethylating agents and histone deacetylase inhibitors (HDACis) are currently under preclinical and clinical investigation. Some of these compounds have been tested and approved for hematological malignancies, but have failed to prove efficacy in solid tumors. Therefore many novel compounds and combination treatments with known chemotherapeutic agents or molecular therapies are currently being tested, aiming to improve the anti-tumoral effects of epigenetic drugs in solid neoplasms. Panobinostat, a novel HDACi, is one promising example that is currently under investigation in combination with Sorafenib in 2 phase I trials for advanced HCC.

\section{Conclusion and future perspectives}

Exhaustive research performed over the past years and the development of new genomic technologies have built the foundation for a better understanding of the molecular pathogenesis of HCC.

Taken all newly generated molecular information into account, testing of molecular targeted therapies in HCC appeared to be a reasonable approach, now also proven true by the widely recognized positive re- 
Table 3

Epigenetic Alterations in Hepatocellular Carcinoma (adapted from Lachenmayer et al. [51] and Hoshida et al. [9])

\begin{tabular}{|c|c|c|c|c|}
\hline & Gene & Gene expression & Epigenetic alteration & Frequency \\
\hline Cell cycle / Apoptosis & $\begin{array}{l}\text { CDKN2A (p16) } \\
\text { PSMD9 (p27) } \\
\text { CDKN2B (p15) } \\
\text { TP53 } \\
\text { CDKN2A (p14) } \\
\text { CASP8 }\end{array}$ & $\begin{array}{l}\text { Decreased } \\
\text { Decreased } \\
\text { Decreased } \\
\text { Decreased } \\
\text { Decreased } \\
\text { Decreased }\end{array}$ & $\begin{array}{l}\text { Hypermethylation } \\
\text { Hypermethylation } \\
\text { Hypermethylation } \\
\text { Hypermethylation } \\
\text { Hypermethylation } \\
\text { Hypermethylation }\end{array}$ & $\begin{array}{l}56-83 \% \\
48 \% \\
43-49 \% \\
14 \% \\
8-20 \%, 25-30 \% \\
72 \%, 43-50 \%\end{array}$ \\
\hline Proliferation/differentiation & $\begin{array}{l}\text { CDH1 (E-Cadherin) } \\
\text { MYC } \\
\text { APC } \\
\text { CDH13 (T-Cadherin) } \\
\text { RAS } \\
\text { SFRP1 } \\
\text { SFRP2 } \\
\text { SFRP5 }\end{array}$ & $\begin{array}{l}\text { Decreased } \\
\text { Increased } \\
\text { Decreased } \\
\text { Decreased } \\
\text { Increased } \\
\text { Decreased } \\
\text { Decreased } \\
\text { Decreased }\end{array}$ & $\begin{array}{l}\text { Hypermethylation } \\
\text { Hypomethylation } \\
\text { Hypermethylation } \\
\text { Hypermethylation / Histone Deacetylation } \\
\text { Hypomehylation } \\
\text { Hypermethylation } \\
\text { Hypermethylation } \\
\text { Hypermethylation }\end{array}$ & $\begin{array}{l}33-67 \% \\
30 \% \\
45-77 \%, 78-91 \%\end{array}$ \\
\hline Growth Factors / Receptors & $\begin{array}{l}\text { IGFBP-3 } \\
\text { RASSF1 } \\
\text { RASSF2 }\end{array}$ & $\begin{array}{l}\text { Decreased } \\
\text { Decreased } \\
\text { Decreased }\end{array}$ & $\begin{array}{l}\text { Hypermethylation } \\
\text { Hypermethylation } \\
\text { Hypermethylation }\end{array}$ & $\begin{array}{l}70 \% \\
85 \%, 54-95 \% \\
6-48 \%\end{array}$ \\
\hline Other & $\begin{array}{l}\text { SOCS1 } \\
\text { SOCS3 } \\
\text { CDH1 } \\
\text { PRDM2 } \\
\text { HIC1 } \\
\text { DCC } \\
\text { RPRM } \\
\text { CACNA1G } \\
\text { RUNX3 } \\
\text { PTGS2 } \\
\text { DLC1 } \\
\text { PGR } \\
\text { ESR1 (ER-alpha) } \\
\text { PYCARD } \\
\text { JNK1 }\end{array}$ & $\begin{array}{l}\text { Decreased } \\
\text { Decreased } \\
\text { Decreased } \\
\text { Decreased } \\
\text { Decreased } \\
\text { Decreased } \\
\text { Decreased } \\
\text { Decreased } \\
\text { Decreased } \\
\text { Decreased } \\
\text { Decreased } \\
\text { Decreased } \\
\text { Decreased } \\
\text { Decreased } \\
\text { Decreased }\end{array}$ & $\begin{array}{l}\text { Hypermethylation } \\
\text { Hypermethylation } \\
\text { Hypermethylation } \\
\text { Hypermethylation } \\
\text { Hypermethylation } \\
\text { Hypermethylation } \\
\text { Hypermethylation } \\
\text { Hypermethylation } \\
\text { Hypermethylation } \\
\text { Hypermethylation } \\
\text { Hypermethylation } \\
\text { H3-K27 methylation } \\
\text { H3-K27 methylation } \\
\text { H3-K9 hypoacetylation and trimethylation } \\
\text { H3 lysines } 4 \text { and } 9 \text { tri-methylation }\end{array}$ & $\begin{array}{l}53-65 \%, 66-67 \% \\
33 \% \\
39-46 \% \\
5-33 \%, 64 \% \\
78-86 \% \\
6-9 \% \\
6-30 \% \\
3-89 \% \\
41-47 \%, 39-82 \% \\
17-46 \% \\
24 \%\end{array}$ \\
\hline DNA repair & $\begin{array}{l}\text { MGMT } \\
\text { GSTP1 }\end{array}$ & $\begin{array}{l}\text { Decreased } \\
\text { Decreased }\end{array}$ & $\begin{array}{l}\text { Hypermethylation } \\
\text { Hypermethylation }\end{array}$ & $\begin{array}{l}22-39 \% \\
41-86 \%\end{array}$ \\
\hline
\end{tabular}

Table 4

Epigenetic alterations with prognostic impact in Hepatocellular Carcinoma

\begin{tabular}{ll}
\hline Epigenetic alteration/signature & Clinical implication \\
\hline Genome wide hypomethylation, degree of hypomethylation & Higher histological grade and larger size of the tumors, poor survival [62,63] \\
Hypermethylation of E-cadherin & Poor survival [72] \\
Methylation profile in surgical margins & Survival [74] \\
DNMTs overexpression & Poor survival [66,67] \\
Agressive phenotype signature & Poor prognosis [73] \\
\hline
\end{tabular}

sults of the multikinase inhibitor sorafenib in advanced HCC [7].

The increasing availability of new technologies to identify molecular alterations in cancer facilitates the important and essential transition from basic researchoriented concepts to translational medicine in our medical centers.

Molecular classification and prognostic signatures will be vital to develop prognostic biomarkers in the future. However, one should keep in mind that those are generated from large retrospective sample series.
To avoid selection biassed and erroneous conclusions, they should always be carefully linked to well classified and selected clinical datasets, otherwise, all this vast amount of information will only add useless complexity.

With an accurate selection of sample datasets, information derived from molecular profiling studies will add valuable information to the daily clinical practice. Although nowadays the ultimate biomarker to define prognosis and to identify treatment responses has not been identified and validated yet, in the near 
future, prognostic genetic or epigenetic signatures and biomarkers are expected to add accuracy to currently used staging systems. This information, once validated, could also be included in the recommendations for clinical trial design guidelines [76], as certain molecular therapies could be tested in selected HCC patients according to their molecular profile to optimize clinical benefits for specific molecular subgroups. In terms of predictors of treatment response, valuable information will emerge soon from clinical trials analyzing new molecular targeted therapies. This will hopefully allow selecting the best treatment for each patient in the future, leading to the expected personalized medicine for each one of our HCC patients.

\section{References}

[1] H.B. El-Serag, Hepatocellular carcinoma: recent trends in the United States, Gastroenterology 127 (2004), S27-S34.

[2] D.M. Parkin, F. Bray, J. Ferlay and P. Pisani, Global cancer statistics, 2002, CA Cancer J Clin 55 (2005), 74-108.

[3] H.B. El-Serag and A.C. Mason, Rising incidence of hepatocellular carcinoma in the United States, $N$ Engl J Med 340 (1999), 745-750.

[4] J.M. Llovet, A. Burroughs and J. Bruix, Hepatocellular carcinoma, Lancet 362 (2003), 1907-1917.

[5] J. Bruix and M. Sherman, Management of hepatocellular carcinoma, Hepatology 42 (2005), 1208-1236.

[6] E.R. Lemmer, S.L. Friedman and J.M. Llovet, Molecular diagnosis of chronic liver disease and hepatocellular carcinoma: the potential of gene expression profiling, Semin Liver Dis 26(4) (Nov 2006), 373-384.

[7] J.M. Llovet, S. Ricci, V. Mazzaferro et al., Sorafenib in advanced hepatocellular carcinoma, N Engl J Med 359 (2008), 378-390.

[8] T. Roskams and M. Kojiro, Pathology of early hepatocellular carcinoma: conventional and molecular diagnosis, Semin Liver Dis 30(1) (Feb 2010), 17-25.

[9] B. Daniele, A. Bencivenga, A.S. Megna and V. Tinessa, Alphafetoprotein and ultrasonography screening for hepatocellular carcinoma, Gastroenterology 127(5 Suppl 1) (Nov 2004 ), S108-S112.

[10] A.I. Gomaa, S.A. Khan, E.L. Leen, I. Waked and S.D. TaylorRobinson, Diagnosis of hepatocellular carcinoma, World $J$ Gastroenterol 15(11) (21 Mar 2009), 1301-1314.

[11] J.A. Marrero, Z. Feng, Y. Wang et al., Alpha-fetoprotein, des-gamma carboxyprothrombin, and lectin-bound alphafetoprotein in early hepatocellular carcinoma, Gastroenterology 137(1) (Jul 2009), 110-118.

[12] M. Capurro, I.R. Wanless, M. Sherman et al., Glypican-3: a novel serum and histochemical marker for hepatocellular carcinoma, Gastroenterology 125(1) (Jul 2003), 89-97.

[13] L. Di Tommaso, G. Franchi, Y.N. Park et al., Diagnostic value of HSP70, glypican 3, and glutamine synthetase in hepatocellular nodules in cirrhosis, Hepatology 45(3) (Mar 2007), $725-734$.

[14] L. Di Tommaso, A. Destro, J.Y. Seok et al., The application of markers (HSP70 GPC3 and GS) in liver biopsies is useful for detection of hepatocellular carcinoma, J Hepatol 50(4) (Apr 2009), 746-754.
[15] J.M. Llovet, C. Brú and J. Bruix, Prognosis of hepatocellular carcinoma: the BCLC staging classification, Semin Liver Dis 19(3) (1999), 329-338.

[16] Y. Hoshida, S. Toffanin, A. Lachenmayer et al., Molecular classification and novel targets in hepatocellular carcinoma: recent advancements, Semin Liver Dis 30(1) (Feb 2010), 3551.

[17] J.S. Lee, I.S. Chu, J. Heo et al., Classification and prediction of survival in hepatocellular carcinoma by gene expression profiling, Hepatology 40(3) (Sep 2004), 667-676.

[18] J.S. Lee, J. Heo, L. Libbrecht et al., A novel prognostic subtype of human hepatocellular carcinoma derived from hepatic progenitor cells, Nat Med 12(4) (Apr 2006), 410-416.

[19] P. Kaposi-Novak, J.S. Lee, L. Gomez-Quiroz, C. Coulouarn, V.M. Factor and S.S. Thorgeirsson, Met-regulated expression signature defines a subset of human hepatocellular carcinomas with poor prognosis and aggressive phenotype, J Clin Invest 116(6) (Jun 2006), 1582-1595.

[20] C. Coulouarn, V.M. Factor and S.S. Thorgeirsson, Transforming growth factor-beta gene expression signature in mouse hepatocytes predicts clinical outcome in human cancer, Hepatology 47(6) (Jun 2008), 2059-2067.

[21] Y. Hoshida, A. Villanueva, M. Kobayashi et al., Gene expression in fixed tissues and outcome in hepatocellular carcinoma, N Engl J Med 359(19) (6 Nov 2008), 1995-2004.

[22] T. Yamashita, M. Forgues, W. Wang et al., EpCAM and alphafetoprotein expression defines novel prognostic subtypes of hepatocellular carcinoma, Cancer Res 68(5) (1 Mar 2008), 1451-1461.

[23] A. Budhu, M. Forgues, Q.H. Ye et al., Prediction of venous metastases, recurrence, and prognosis in hepatocellular carcinoma based on a unique immune response signature of the liver microenvironment, Cancer Cell 10(2) (Aug 2006), 99-111.

[24] H. Katoh, H. Ojima, A. Kokubu et al., Genetically distinct and clinically relevant classification of hepatocellular carcinoma: putative therapeutic targets, Gastroenterology 133(5) (Nov 2007), 1475-1486.

[25] S. Boyault, D.S. Rickman, A. de Reyniès et al., Transcriptome classification of HCC is related to gene alterations and to new therapeutic targets, Hepatology 45(1) (Jan 2007), 42-52.

[26] D.Y. Chiang, A. Villanueva, Y. Hoshida ey al., Focal gains of VEGFA and molecular classification of hepatocellular carcinoma, Cancer Res 68(16) (15 Aug 2008), 6779-6788.

[27] Y. Kurokawa, R. Matoba, I. Takemasa et al., Molecular-based prediction of early recurrence in hepatocellular carcinoma, $J$ Hepatol 41(2) (Aug 2004), 284-291.

[28] H.G. Woo, E.S. Park, J.H. Cheon et al., Gene expression-based recurrence prediction of hepatitis B virus-related human hepatocellular carcinoma, Clin Cancer Res 14(7) (1 Apr 2008), 2056-2064.

[29] S.M. Wang, L.L. Ooi and K.M. Hui, Identification and validation of a novel gene signature associated with the recurrence of human hepatocellular carcinoma, Clin Cancer Res 13(21) (1 Nov 2007), 6275-6283.

[30] S. Yoshioka, I. Takemasa, H. Nagano et al., Molecular prediction of early recurrence after resection of hepatocellular carcinoma, Eur J Cancer 45(5) (Mar 2009), 881-889.

[31] N. Iizuka, M. Oka, H. Yamada-Okabe et al., Oligonucleotide microarray for prediction of early intrahepatic recurrence of hepatocellular carcinoma after curative resection, Lancet 361(9361) (15 Mar 2003), 923-929.

[32] Y. Hoshida, S.M. Nijman, M. Kobayashi et al., Integrative transcriptome analysis reveals common molecular subclasses 
of human hepatocellular carcinoma, Cancer Res 69(18) (15 Sep 2009), 7385-7392.

[33] B. Mínguez, Y. Hoshida, A. Villanueva et al., Gene-expression signature of vascular invasion in hepatocullular carcinoma, $J$ Hepato (3 Apr 2011).

[34] A. Villanueva, P. Newell, D.Y. Chiang et al., Molecular and clinical characterization of hepatocellular carcinoma with progenitor cell markers, Hepatology 48(4 Suppl) (2008), 361A.

[35] Y. Kurokawa, R. Matoba, I. Takemasa et al., Molecular-based prediction of early recurrence in hepatocellular carcinoma, $J$ Hepatol 41(2) (Aug 2004), 284-291.

[36] S. Cairo, C. Armengol, A. De Reynies et al., Hepatic stemlike phenotype and interplay of Wnt/beta-catenin and Myc signaling in aggressive childhood liver cancer, Cancer Cell 14(6) (9 Dec 2008), 471-484.

[37] P. Laurent-Puig, P. Legoix, O. Bluteau et al., Genetic alterations associated with hepatocellular carcinomas define distinct pathways of hepatocarcinogenesis, Gastroenterology 120(7) (Jun 2001), 1763-1773.

[38] A. Villanueva, B. Minguez, A. Forner et al., Hepatocellular carcinoma: novel molecular approaches for diagnosis, prognosis, and therapy, Апnu Rev Med 61 (2010), 317-328.

[39] D.P. Bartel, MicroRNAs: genomics, biogenesis, mechanism, and function, Cell 116(2) (23 Jan 2004), 281-297.

[40] G.A. Calin and C.M. Croce, MicroRNA signatures in human cancers, Nat Rev Cancer 6 (2006), 857-866.

[41] Y. Murakami, T. Yasuda, K. Saigo et al., Comprehensive analysis of microRNA expression patterns in hepatocellular carcinoma and non-tumorous tissues, Oncogene 25(17) (20 Apr 2006), 2537-2545.

[42] C.Z. Chen, MicroRNAs as oncogenes and tumor suppressors, N Engl J Med 353(17) (27 Oct 2005), 1768-1771.

[43] J. Lu, G. Getz, E.A. Miska et al., MicroRNA expression profiles classify human cancers, Nature 435(7043) (9 Jun 2005), 834-838.

[44] S. Toffanin, Y. Hoshida, A. Lachenmayer et al., MicroRNAbased classification of hepatocellular carcinoma and oncogenic role of miR-517a. Gastroenterology 140(5) (May 2011), 1618-1628.

[45] G.A. Calin, M. Ferracin, A. Cimmino et al., A MicroRNA signature associated with prognosis and progression in chronic lymphocytic leukemia, N Engl J Med 353 (2005), 1793-1801.

[46] A.J. Schetter, S.Y. Leung, J.J. Sohn et al., MicroRNA expression profiles associated with prognosis and therapeutic outcome in colon adenocarcinoma, JAMA 299 (2008), 425-436.

[47] S.F. Tavazoie, C. Alarcon, T. Oskarsson et al., Endogenous human microRNAs that suppress breast cancer metastasis, $\mathrm{Na}$ ture 451 (2008), 147-152.

[48] L. Gramantieri, F. Fornari, M. Ferracin et al., MicroRNA-221 targets Bmf in hepatocellular carcinoma and correlates with tumor multifocality, Clin Cancer Res 15(16) (15 Aug 2009), 5073-5081.

[49] W. Li, L. Xie, X. He et al., Diagnostic and prognostic implications of microRNAs in human hepatocellular carcinoma, Int J Cancer 123(7) (1 Oct 2008), 1616-1622.

[50] X. Zhang, S. Liu, T. Hu, S. Liu, Y. He and S. Sun, Up-regulated microRNA-143 transcribed by nuclear factor kappa B enhances hepatocarcinoma metastasis by repressing fibronectin expression, Hepatology 50(2) (Aug 2009), 490-499.

[51] S.R. Viswanathan, J.T. Powers, W. Einhorn et al., Lin28 promotes transformation and is associated with advanced human malignancies, Nat Genet 41(7) (Jul 2009), 843-848.
[52] J. Ji, J. Shi, A. Budhu et al., MicroRNA expression, survival, and response to interferon in liver cancer, $N$ Engl J Med 361(15) (8 Oct 2009), 1437-1447.

[53] C. Coulouarn, V.M. Factor, J.B. Andersen, M.E. Durkin and S.S. Thorgeirsson, Loss of miR-122 expression in liver cancer correlates with suppression of the hepatic phenotype and gain of metastatic properties, Oncogene 28(40) (8 Oct 2009), 35263536.

[54] A. Budhu, H.L. Jia, M. Forgues et al., Identification of metastasis-related microRNAs in hepatocellular carcinoma, Hepatology 47 (2008), 897-907.

[55] J. Jiang, Y. Gusev, I. Aderca et al., Association of MicroRNA expression in hepatocellular carcinomas with hepatitis infection, cirrhosis, and patient survival, Clin Cancer Res 14 (2008), 419-427.

[56] F. Meng, R. Henson, H. Wehbe-Janek, K. Ghoshal, S.T. Jacob and T. Patel, MicroRNA-21 regulates expression of the PTEN tumor suppressor gene in human hepatocellular cancer, Gastroenterology 133(2) (Aug 2007), 647-658.

[57] F. Fornari, L. Gramantieri, M. Ferracin et al., MiR-221 controls CDKN1C/p57 and CDKN1B/p27 expression in human hepatocellular carcinoma, Oncogene 27(43) (25 Sep 2008), 5651-5661.

[58] J. Kota, R.R. Chivukula, K.A. O'Donnell et al., Therapeutic microRNA delivery suppresses tumorigenesis in a murine liver cancer model, Cell 137(6) (12 Jun 2009), 1005-1017.

[59] V. Wang and W. Wu, MicroRNA-based therapeutics for cancer, BioDrugs 23(1) (2009), 15-23.

[60] J. Krutzfeldt, N. Rajewsky, R. Braich et al., Silencing of microRNAs in vivo with 'antagomirs', Nature 438 (2005), 685689.

[61] E. Ballestar and M. Esteller, Epigenetic gene regulation in cancer, Adv Genet 61 (2008), 247-267.

[62] C.H. Lin, S.Y. Hsieh, I.S. Sheen et al., Genome-wide hypomethylation in hepatocellular carcinogenesis, Cancer Res 61(10) (15 May 2001), 4238-4243.

[63] D.F. Calvisi, S. Ladu, A. Gorden et al., Mechanistic and prognostic significance of aberrant methylation in the molecular pathogenesis of human hepatocellular carcinoma, J Clin Invest 117(9) (Sep 2007), 2713-2722.

[64] M. Nishigaki, K. Aoyagi, I. Danjoh et al., Discovery of aberrant expression of R-RAS by cancer-linked DNA hypomethylation in gastric cancer using microarrays, Cancer Res 65(6) (15 Mar 2005), 2115-2124.

[65] A. Lachenmayer, C. Alsinet, C.Y. Chang and J.M. Llovet, Molecular approaches to treatment of hepatocellular carcinoma, Dig Liver Dis 42(Suppl 3) (Jul 2010), S264-S272.

[66] B.K. Oh, H. Kim, H.J. Park et al., DNA methyltransferase expression and DNA methylation in human hepatocellular carcinoma and their clinicopathological correlation, Int J Mol Med 20 (2007), 65-73.

[67] Y. Saito, Y. Kanai, T. Nakagawa et al., Increased protein expression of DNA methyltransferase (DNMT) 1 is significantly correlated with the malignant potential and poor prognosis of human hepatocellular carcinomas, Int J Cancer 105 (2003), 527-532.

[68] N. Nishida, T. Nagasaka, T. Nishimura, I. Ikai, C.R. Boland and A. Goel, Aberrant methylation of multiple tumor suppressor genes in aging liver, chronic hepatitis, and hepatocellular carcinoma, Hepatology 47(3) (Mar 2008), 908-918.

[69] C. Zhang, H. Li, G. Zhou et al., Transcriptional silencing of the TMS1/ASC tumour suppressor gene by an epigenetic mechanism in hepatocellular carcinoma cells, J Pathol 212 (2007), 134-142. 
[70] Y. Kondo, L. Shen, S. Suzuki et al., Alterations of DNA methylation and histone modifications contribute to gene silencing in hepatocellular carcinomas, Hepatol Res 37 (2007), 974-83.

[71] M. Esteller, P.G. Corn, S.B. Baylin and J.G. Herman, A gene hypermethylation profile of human cancer, Cancer Res $\mathbf{6 1}$ (2001), 3225-3229.

[72] S. Lee, H.J. Lee, J.H. Kim, H.S. Lee, J.J. Jang and G.H. Kang, Aberrant $\mathrm{CpG}$ island hypermethylation along multistep hepatocarcinogenesis, Am J Pathol 163 (2003), 1371-1378.

[73] A. Lachenmayer, Y. Hoshida, S. Toffanin et al., DNA hypermethylation subclass of hepatocellular carcinoma associated with aggressive phenotype, Hepatology 52(4 suppl) (2010), 327A.

74] C. Lou, Z. Du, B. Yang, Y. Gao, Y. Wang and S. Fang, Aberrant DNA methylation profile of hepatocellular carcinoma and surgically resected margin, Cancer Sci 100 (2009), 996-1004.

[75] C.B. Yoo and P.A. Jones, Epigenetic therapy of cancer: past, present and future, Nat Rev Drug Discov 5(1) (Jan 2006), 37-50.

[76] J.M. Llovet, A.M. Di Bisceglie, J. Bruix et al., Design and endpoints of clinical trials in hepatocellular carcinoma, $J$ Natl Cancer Inst 100(10) (21 May 2008), 698-711. 


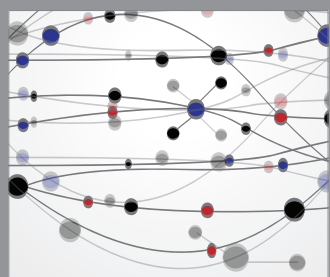

The Scientific World Journal
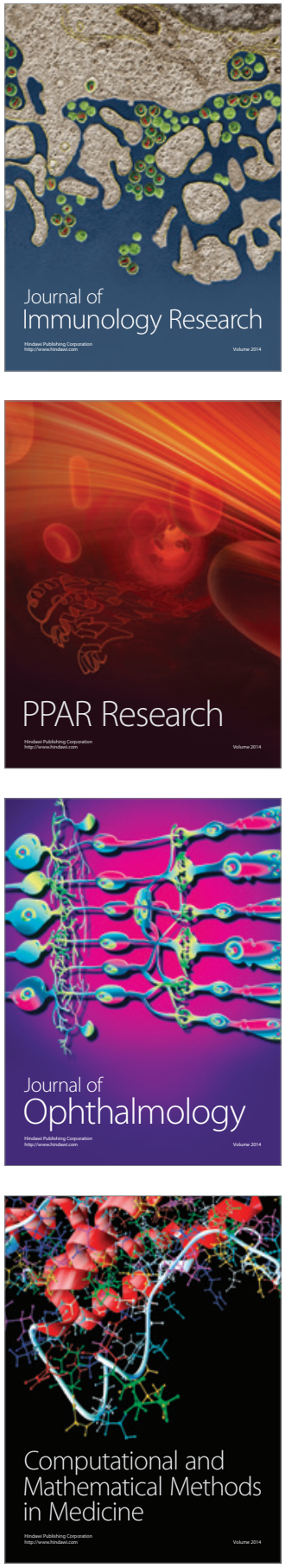

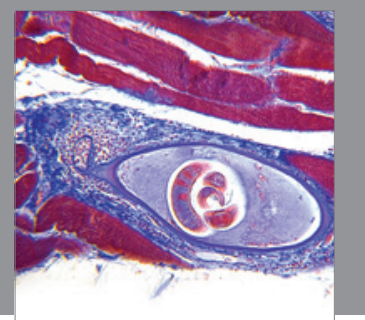

Gastroenterology

Research and Practice
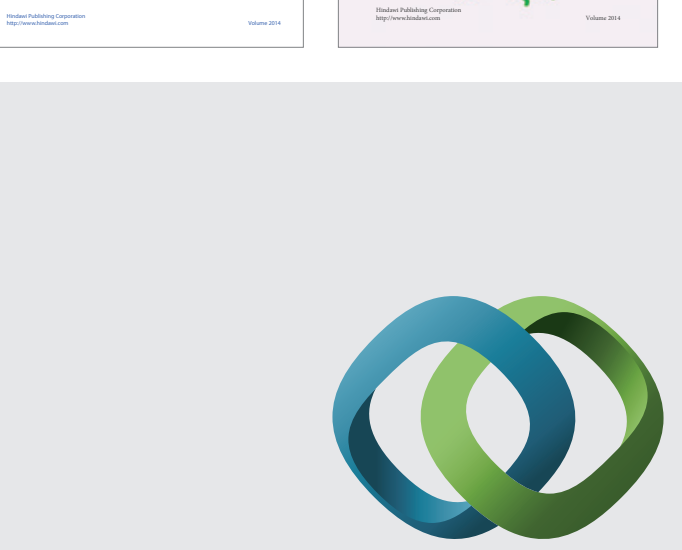

\section{Hindawi}

Submit your manuscripts at

http://www.hindawi.com
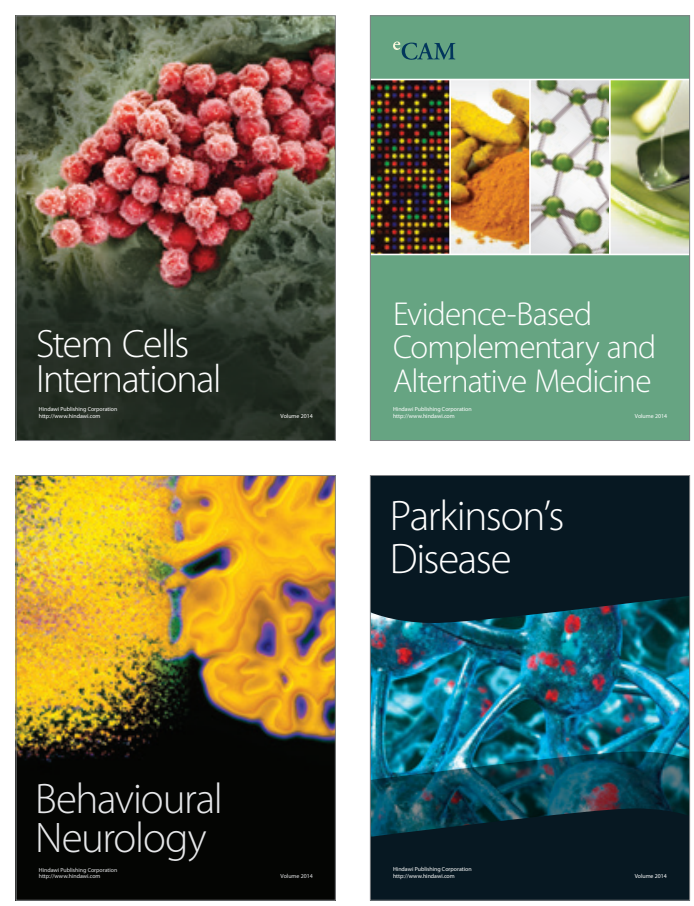

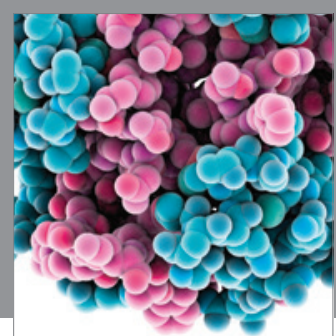

Journal of
Diabetes Research

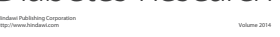

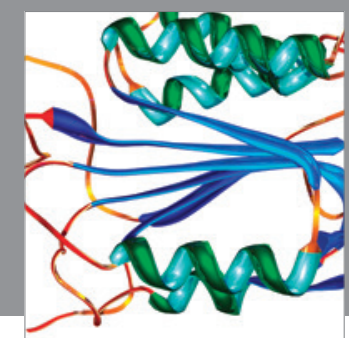

Disease Markers
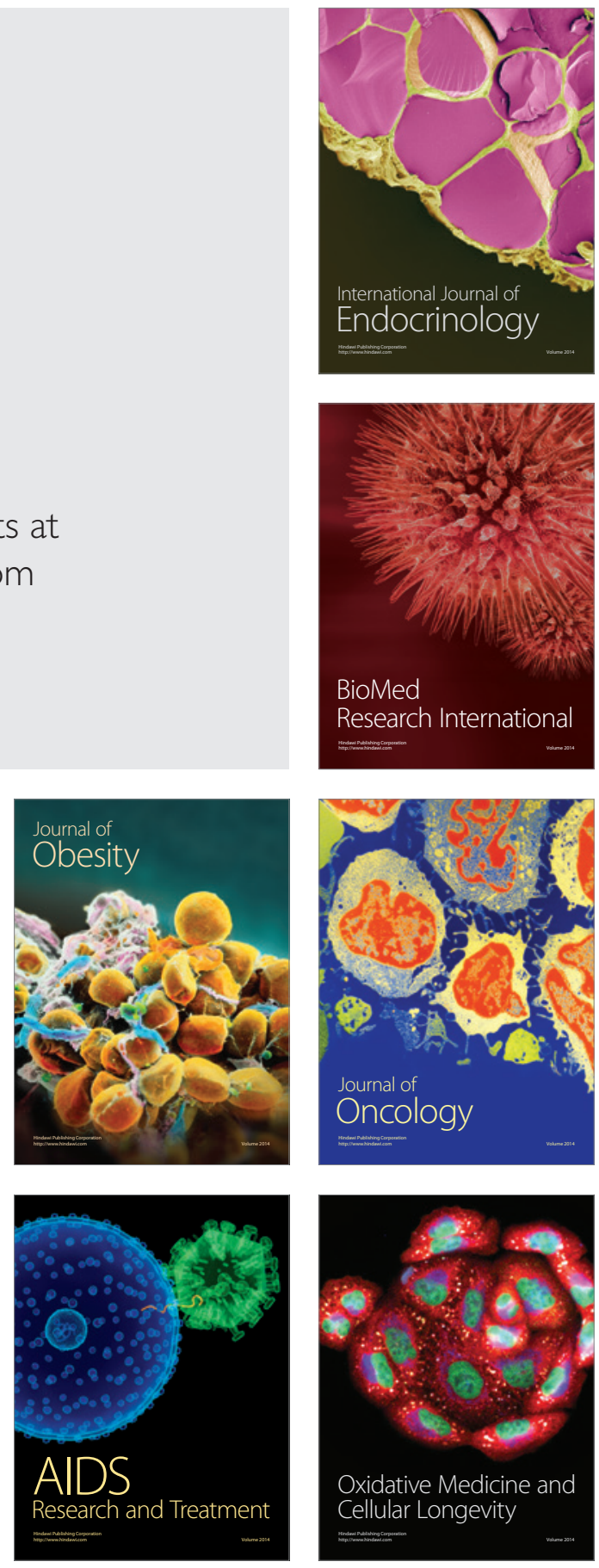